\title{
On-chip Hybrid Integration of Silicon Nitride Microdisk With Colloidal Quantum Dots
}

\author{
Weiqiang Xie, ${ }^{1,2,}$ Yunpeng Zhu, ${ }^{1,2}$ Tangi Aubert, ${ }^{2,3}$ Zeger Hens, ${ }^{2,3}$ Edouard Brainis, ${ }^{2,3}$ and Dries Van Thourhout ${ }^{1,2}$ \\ ${ }^{1}$ Photonics Research Group, Department of Information Technology, Ghent University-IMEC, Gent B-9000, Belgium \\ ${ }^{2}$ Center for Nano and Biophotonics, Ghent University, B-9000 Ghent, Belgium \\ ${ }^{3}$ Physics and Chemistry of Nanostructures, Ghent University, B-9000 Ghent, Belgium \\ *weiqiang.xie@intec.ugent.be
}

\begin{abstract}
We report on the fabrication of on-chip freestanding silicon nitride microdisks hybridly integrated with embedded colloidal quantum dots. An efficient coupling of quantum dot emission to resonant disk modes in the visible range is demonstrated.
\end{abstract}

Keywords - silicon nitride; microdisk; colloidal quantum dots; on-chip integration

\section{INTRODUCTION}

Given its moderate optical index $(\sim 2.0)$, broad operating wavelength range and low-loss, silicon nitride $(\mathrm{SiN})$ photonics is a promising platform for various applications including integrated photonic circuits [1], nonlinear optics [2], and onchip biosensing [3]. On the other hand, because of its dielectric nature, $\mathrm{SiN}$ devices have thus far been limited to passive functionalities and an optically active $\mathrm{SiN}$ photonics platform is highly desired. One of the possible choices is to combine gain material with $\mathrm{SiN}$ building blocks such as waveguides and resonators $[4,5]$ for making active devices e.g. a laser or a single photon emitter. As a novel class of materials, colloidal quantum dots (QDs), with their efficient and broadly tunable luminescence, are an ideal candidate for integration with $\mathrm{SiN}$ photonics. An efficient integration process should preserve the optical properties of the QDs and allow realization of low-loss SiN circuits. More importantly, to enable effective coupling between the QD emission and waveguide or cavity modes, these modes should maximumly overlap with the QD emitters. In practice, it is also important that these active SiN-QD devices are combined with low loss passive access waveguides that allow combining multiple devices or coupling the generated light of chip. In this work, we developed a fully inorganic, low-loss SiN-QD hybrid photonics platform by using low-temperature $\left(270^{\circ} \mathrm{C}\right)$ plasma enhanced chemical vapor deposition (PECVD) and optimized reactive ion etching (RIE) processes. Based on this platform, we designed and fabricated free-standing SiN microdisks with colloidal QDs embedded inside, and coupled them vertically with on-chip SiN bus waveguides. Efficient coupling of QD photoluminescence (PL) to disk modes is experimentally demonstrated. Furthermore, the coupling strength and selectivity for different order modes can be controlled by tuning the parameters of the geometric configuration. These high-performance active SiN-QD devices open up new opportunities for both QD-based quantum optics and SiNbased photonic applications.

\section{DESIGN AND FABRICATION}

The design of the SiN-QD disk with vertically coupled waveguide is shown in Fig. 1. The bus waveguide is defined on a silicon wafer with a $3 \mu \mathrm{m} \mathrm{SiO}_{2}$ layer and is also sideways embedded in $\mathrm{SiO}_{2}$. An amorphous silicon (aSi) pillar supports the SiN disk with the embedded QD layer. The height of the aSi pillar determines the vertical coupling gap between the disk and the waveguide. The undercut distance $d$ is chosen sufficiently large to prevent leakage of the fundamental transverse electric (TE) modes to the aSi pillar. To tune the coupling strength, the horizontal offset between the center of the bus waveguide and the edge of the disk is adjusted from $400 \mathrm{~nm}$ to $+400 \mathrm{~nm}$, with positive offsets denoting a bus waveguide closer towards the centre of the disk.

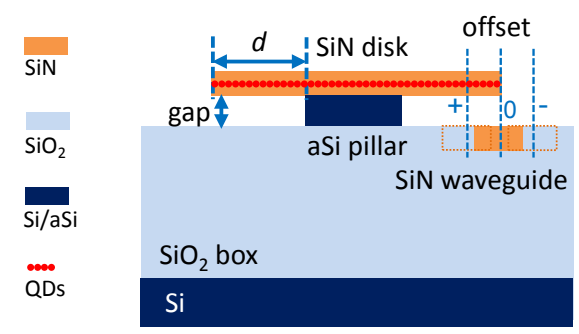

Fig. 1. Cross-sectional schematics of device design.

From finite-difference time-domain simulations, we choose a height of $\sim 135 \mathrm{~nm}$ for the bus waveguide, guaranteeing single mode operation at a wavelength of $625 \mathrm{~nm}$, the peak PL wavelength of the QDs employed. The disk height was set at $\sim 180 \mathrm{~nm}$ to ensure low-loss for the TE-like disk modes. The width of the bus waveguide and the diameter of the SiN disk were varied to study their influence on the disk-waveguide coupling. The vertical coupling gap was fixed at $\sim 200 \mathrm{~nm}$. Note that there are two main advantages to our vertical coupling configuration. First, it allows for an on-chip waveguide-coupled free-standing disk and therefore to achieve high quality (Q) whispering gallery modes (WGM) with low radiation loss, even for relatively small diameters. Second, because of the separation of the fabrication steps for waveguide and disk, we can integrate the QDs exclusively in the disk and not in the access waveguides. This allows to 
study the coupling of the QD emission to disk modes without background interference, which would not be possible if also the waveguides contain QDs as would be unavoidable in a lateral coupling scheme.

The fabrication starts with the deposition of a $\sim 135 \mathrm{~nm}$ PECVD SiN layer onto a wafer with a $3 \mu \mathrm{m}$ thermal $\mathrm{SiO}_{2}$ box layer. Next the bus waveguide is patterned using contact lithography and RIE etching to form a strip waveguide. Both the contact lithography and the RIE process were optimized to be able to define a low-loss waveguide supporting only a single TE-like mode at the operating wavelength. This ensures only the TE-like disk modes are coupled out efficiently. After waveguide fabrication, a $800 \mathrm{~nm}$ PECVD $\mathrm{SiO}_{2}$ cladding is deposited and a chemical mechanical polishing step is applied down the top of waveguide layer to planarize the surface, as shown in Fig. 1. Next, the vertical coupling gap is defined by depositing a $200 \mathrm{~nm}$ thick PECVD aSi layer. The SiN-QD composite layer is prepared by first depositing a $\sim 90 \mathrm{~nm} \mathrm{SiN}$ layer, then spin-coating a nearly monolayer of QDs, and then depositing another $\sim 80 \mathrm{~nm} \mathrm{SiN}$ layer on top of the QDs. These latter ones consist in $10 \mathrm{~nm} \mathrm{CdSe} / \mathrm{CdS}$ core/shell QDs prepared according to the "flash" synthesis procedure [6]. The disk is defined using the same process as used for the waveguides. Finally an alkaline based wet etch is utilized to undercut the aSi and to realize the free-standing SiN-QD disk schematically depicted in Fig. 1. The microscope and SEM pictures of Fig. 2(a-c) show the designed structure is accurately realized.

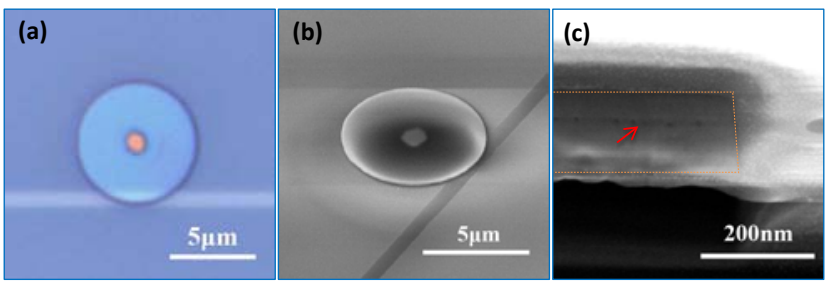

Fig. 2. (a) Optical and (b) scanning electron microscope images of one fabricated device with a $7 \mu \mathrm{m}$ diameter disk. (c) Cross section of the etched profile of disk with the red arrow indicating the embedded QDs.

\section{CHARACTERIZATION RESUlTS}

We characterized the PL spectrum of the fabricated devices. A $400 \mathrm{~nm}$ pulsed femtosecond laser was used for pumping the disk from the top and the PL-signal was collected from the cleaved facet of the bus waveguide using microlensed fibers. In Fig. 3, we show the normalized PL spectra of disks with various radii coupled to a $\sim 500 \mathrm{~nm}$ wide bus waveguide with an offset of about $-160 \mathrm{~nm}$. The envelope of these PL spectra arises both from the wavelength-dependent emission of the QDs (inset of (a)) and from the wavelengthdependent disk-waveguide coupling. The spectra clearly show the TE WGM modes and the background PL signal is very strongly suppressed. This can be attributed to the enhanced interaction between the QDs and the disk modes and the efficient coupling of the disk modes to the access waveguide. Since the location of the QDs is aligned with the maximum of the light field in the disk we can boost the interaction between them and improve the coupling efficiency of the QD emission into the disk modes. In Fig. 3(a-d), we also denote the Q- factors of the resonances around $625 \mathrm{~nm}$ obtained by fitting a Lorentzian. We obtain a Q of 1160 with a free spectral range (FSR) of $\sim 8.8 \mathrm{~nm}$ for the $3.5 \mu \mathrm{m}$ radius disk and a $\mathrm{Q}$ of 5740 with a $\sim 3.0 \mathrm{~nm}$ FSR for the $10.0 \mu \mathrm{m}$ radius disk. Note that characterization of the modes with $Q$ above $\sim 4000$ is already limited by the $0.05 \mathrm{~nm}$ resolution of the spectrometer we used and an even higher actual $\mathrm{Q}$ is therefore expected for the 10.0 $\mu \mathrm{m}$ radius disk. In Fig. 3(c) and even more so in Fig. 3(d), the second order TE mode family also starts to appear. By increasing the width of the bus waveguide, we can reduce the undesired coupling of the second order TE modes, as shown in the inset of Fig. 3(d). The coupling strength for a certain mode family can also be tuned by changing the offset (not shown here), illustrating further the advantages of the vertical coupling scheme.
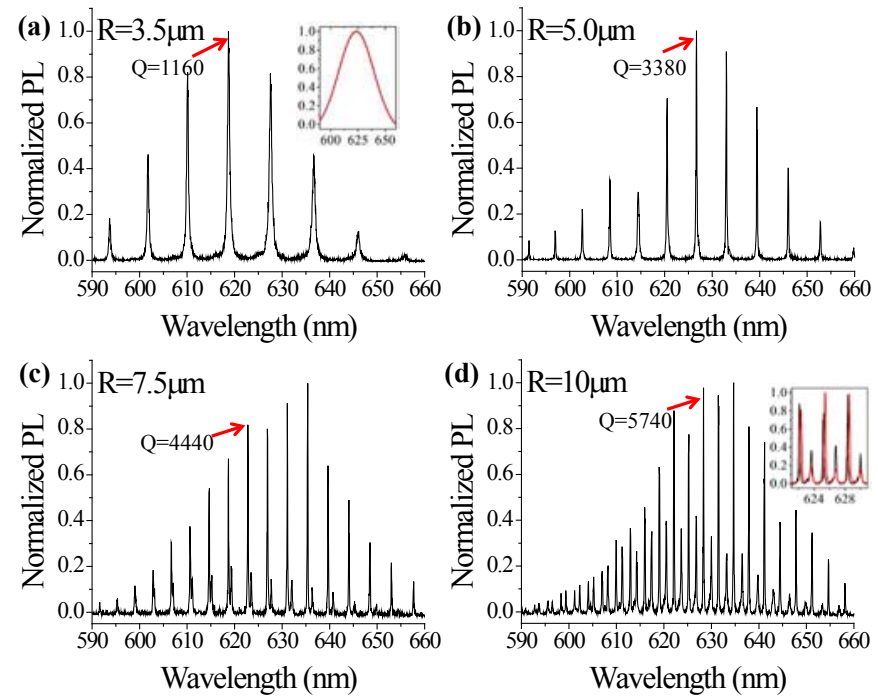

Fig. 3. (a-d) Normalized PL spectra of the devices with different radius disks coupled to $\sim 500 \mathrm{~nm}$ wide waveguides under an offset of around $-160 \mathrm{~nm}$. Red arrows designate the first order TE modes around $625 \mathrm{~nm}$ together with the fitted Q. The inset in (a) shows the PL from slab waveguide for reference. The inset in (d) presents two PL spectra coupled out from different bus waveguides with widths of $\sim 500 \mathrm{~nm}$ and $\sim 670 \mathrm{~nm}$ for the black and red curves, respectively.

\section{CONCLUSION}

We designed and fabricated on-chip free-standing $\mathrm{SiN}$ microdisks hybridly integrated with embedded colloidal QDs. Efficient coupling of QD emission to disk resonance modes has been demonstrated in the visible range. These active SiN devices offer a promising platform for both QD-based quantum optics and the SiN photonic community.

\section{ACKNOWLEDGMENT}

The authors acknowledge the ERC-ULPPIC and the IAP Photon@BE projects for financial support.

\section{REFERENCES}

[1] Q. Li et al., Opt. Express 21, 18236 (2013).

[2] D. J. Moss et al., Nat. Photonics 7, 597 (2013).

[3] X. G. Tu et al., Opt. Express 20, 2640 (2012).

[4] B. De Geyter et al., Appl. Phys. Lett. 101 (2012).

[5] S. Gupta, and E. Waks, Opt. Express 21, 29612 (2013).

[6] M. Cirillo et al., Chem. Mater. 26, 1154 (2014). 